Proceedings of the International School and Conference on Optics and Optical Materials, ISCOM07, Belgrade, Serbia, September 3-7, 2007

\title{
NIST Cesium Fountains - Current Status and Future Prospects
}

\author{
S.R. Jefferts*, T.P. Heavner, T.E. Parker \\ AND J.H. SHIRLEY \\ NIST - Time and Frequency Division
}

325 Broadway, Boulder, CO 80305, USA

\begin{abstract}
We review the current status of the U.S. Primary Frequency Standard, NIST-F1. NIST-F1 is a laser-cooled cesium fountain based frequency standard with an inaccuracy of less than $\delta f / f \leq 5 \times 10^{-16}$; limited mainly by the radiation field in the room-temperature fountain (blackbody shift). NIST-F1 is one of the best cesium fountains currently contributing to international atomic time, but has reached a point that it is impractical to improve its accuracy substantially. Therefore we are building a new fountain, imaginatively named NIST-F2, with a cryogenic (77 K) Ramsey interrogation zone that lowers the blackbody shift by several orders of magnitude. NIST-F2 is currently undergoing final assembly, and we will discuss our planned (hoped for) performance, which includes frequency inaccuracy of $\delta f / f<1 \times 10^{-16}$.
\end{abstract}

PACS numbers: 06.20.Fn, 32.30.Bv, 32.30-r, 32.10.Fn, 32.80.Pj

\section{Fountain frequency standards}

In the International System of units (SI) the second is defined as the duration of 9,192,631,770 periods of the radiation corresponding to the transition between two hyperfine levels of the ground state of the cesium-133 atom. The definition was later expanded to include the phrase "This definition refers to a cesium atom at rest at a temperature of $0 \mathrm{~K}$ ". Clearly what is being defined is, in fact, the frequency of that transition as being $9.192631770 \mathrm{GHz}$. Primary frequency standards are an attempt to realize this definition and, where possible, correct for inadequacies in that realization. This document will describe the current state of the art in realizing that definition and show some of the paths forward in attempting to improve our primary frequency standards to even higher accuracies.

The basic operation of a fountain based frequency standard can be boiled down to a few discrete steps. See Fig. 1 for a schematic diagram of a typical cesium

*corresponding author; e-mail: jefferts@boulder.nist.gov 


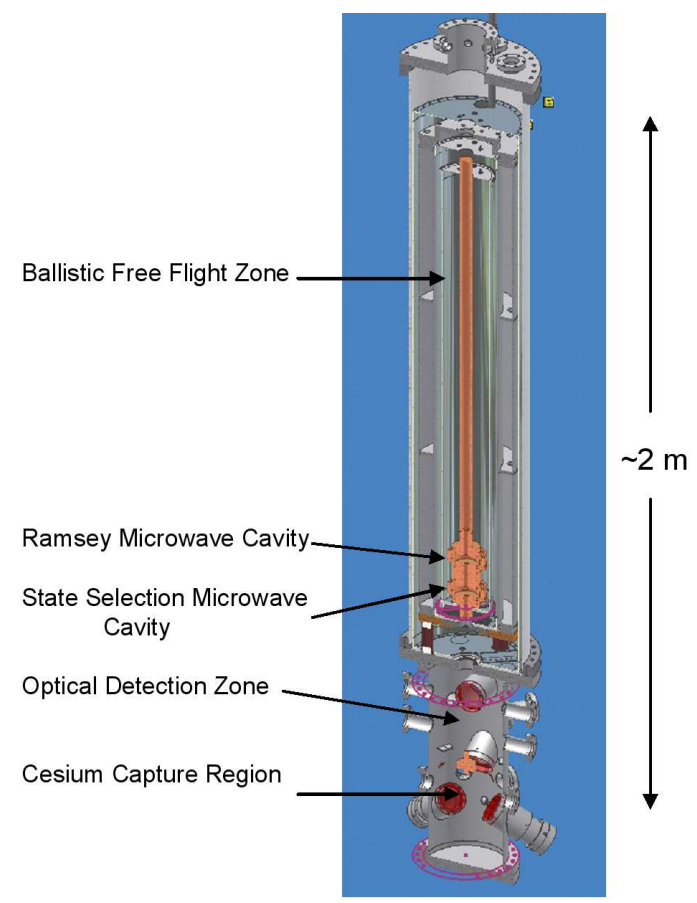

Fig. 1. A simplified view of the "physics package" of a cesium fountain. This includes the atom capture region, detection region, state-selection cavity, Ramsey cavity, and drift tube. These devices are typically about $2 \mathrm{~m}$ tall.

fountain. First a sample of cesium atoms (typically $\approx 10^{7}$ atoms) is captured in either an optical molasses or a magneto-optical trap (MOT), next the sample of cesium atoms is launched vertically by detuning the laser beams in the downward direction to a lower frequency and the laser beams in the upward direction to a higher frequency $(\approx 1(\mathrm{~m} / \mathrm{s}) / \mathrm{MHz}$ detuning). The launched ball of atoms is subject to a final laser cooling stage immediately after launch, which leaves the "ball" of atoms with a center of mass velocity determined by the detuning of the laser beams, and a random velocity on the order of $1 \mathrm{~cm} / \mathrm{s}$, determined by the quality of this final "post-cooling" stage. The launched ball of cesium atoms is typically all in the $F=4$ state but more or less evenly distributed throughout the $m_{f}=-4,-3, \ldots, 4$ levels. Because the $m_{f}=0$ to $m_{f}=0(|3,0\rangle \leftrightarrow|4,0\rangle)$ transition is, to first order, insensitive to magnetic field this transition is used as the clock transition. The launched sample of atoms is therefore typically "state-selected" in a microwave cavity where the $|4,0\rangle$ atoms in the ball are driven into the $|3,0\rangle$ state and the remaining $F=4$ are atoms $\left(m_{f} \neq 0\right)$ removed with an optical blast. This leaves a pure $|3,0\rangle$ sample of atoms still traveling upward on their ballistic trajectory. This $|3,0\rangle$ sample of atoms next encounters a second microwave cavity tuned to the $9.193 \mathrm{GHz}$ transition from $|3,0\rangle$ to $|4,0\rangle$. Inside this cavity (known as the 
Ramsey cavity) the atoms are driven into a coherent superposition between the $|3,0\rangle$ and $|4,0\rangle$ states. The atoms continue upwards, reach apogee, and return to the Ramsey cavity traveling in the opposite direction as their previous passage through the cavity. Depending on the relative phase of the coherent superposition and the microwave field in the cavity the atoms are driven toward either the $|3,0\rangle$ state or the $|4,0\rangle$ state. Finally the ball of atoms must be sampled to determine what fraction of the atoms made the transition from the $|3,0\rangle$ state to the $|4,0\rangle$ state. This information is used to tune the frequency of the microwaves to reflect the $9.192631770 \mathrm{GHz}$ transition frequency of the cesium atoms. That is, when the frequency is correct, the transition probability is maximized. This peak in the transition probability is then utilized to "steer" the microwave frequency. The process typically takes about $1 \mathrm{~s}$. The sequence of cool, launch, interrogate and measure sequence takes about $1 \mathrm{~s}$ and is repeated many, typically $\approx 10^{6}$, times and the results averaged in order to calibrate the rate of a local clock, typically a hydrogen maser. This style of measurement, known as Ramsey interferometry, is used in all fountain frequency standards. Much more detailed information can be found in $[1-4]$.

\section{Frequency shifts - statistical}

As mentioned above, the result of a measurement of the rate of a local clock with NIST-F1 is the result of several weeks of operation and contains something like $10^{6}$ discrete measurements. Typically the Allan variance [5] is used to examine the statistics of the various measurements to ensure that the resulting average, along with its uncertainty, is a valid statement of the statistical uncertainty of the total measurement process. The precision with which a frequency can be measured in a fountain is a function of the signal to noise ratio $(\mathrm{S} / \mathrm{N})$ in a single measurement along with the number of single measurements that are combined to get the final result. In a well designed fountain the $\mathrm{S} / \mathrm{N}$ ratio is determined by two factors, the number of atoms and the noise in the local oscillator. When using a small number of atoms the noise should be set by the quantum projection noise (QPN) [6] where $S / N \propto 1 / \sqrt{N}$ ( $N$ is the number of atoms) (this looks like shot noise in the number of atoms). As the number of atoms is increased, the fountain precision for one measurement generally improves until it is limited by noise in the local oscillator at the $\delta f / f \approx 1-2 \times 10^{-13}$ level, although by using a cryogenic oscillator as the LO, the group in Paris at SYRTE have managed to reduce this to the $\delta f / f \approx 3 \times 10^{-14}$ level. When normal Gaussian (white) noise processes (such as QPN) are dominant, the uncertainty varies as $1 / \sqrt{N}$, where $N$ is the number of discrete measurements. Under these conditions the Allan variance has a slope of $1 / \sqrt{\tau}$ where $\tau$ is the duration (total time) of the measurement; a typical Allan variance plot from NIST-F1 is shown in Fig. 2, and from this it can be seen that the results of the measurement are consistent with white-noise processes at all measurement times (the slope is consistent with $1 / \sqrt{\tau}$ for all $\tau$ ). Moreover, 


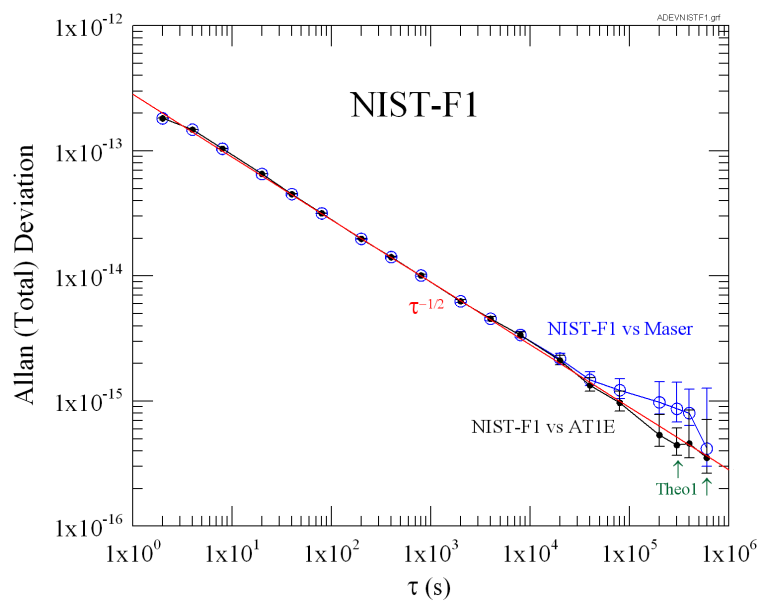

Fig. 2. The Allan deviation from a recent measurement campaign of NIST-F1. As can be seen there are no significant deviations from a $1 / \sqrt{\tau}$ behavior in the case of NIST-F1 vs AT1E (AT1E is a timescale generated from the combined output of a number of hydrogen masers), as should be expected for a primary frequency standard. The deviation from $1 / \sqrt{\tau}$ behavior in NIST-F1 vs. maser is due to the maser and is expected.

Fig. 2 shows that the frequency uncertainty with a single frequency measurement is around $\delta f / f \approx 2 \times 10^{-13}$; after $10^{6}$ measurements the uncertainty is reduced to a level of $\delta f / f \approx 2 \times 10^{-13} / \sqrt{10^{6}}=2 \times 10^{-16}$. This is typical of the statistical uncertainty resulting from the measurement noise in NIST-F1.

\section{Frequency shifts - systematic}

The definition of the second involves the hyperfine splitting of a cesium atom unperturbed by its environment. Unfortunately, in the laboratory, there exists a host of possible perturbations of the atom's structure. Some are planned as a result of the experimental configuration, while others, generally unplanned, can be the object of extensive searches for unexplained instabilities of the fountain. Examples of planned perturbations include the facts that, one, a carefully controlled magnetic field is applied to the atom to define the quantization axis and, two, the apparatus is temperature stabilized around $40^{\circ} \mathrm{C}$ to keep frequency of the Ramsey microwave cavity resonance at the same frequency as the cesium hyperfine structure. Unplanned systematic frequency shifts generally include such things as uncontrolled optical and microwave fields appearing in parts of the apparatus where they should not be. We will first examine several of the known biases and then some of the unplanned biases. What is critical in this context is not the magnitude of any individual bias but rather its associated uncertainty. For example, the magnetic field causes a bias of some $\delta f / f \approx 2 \times 10^{-14}$; but because we measure this value to high precision throughout a calibration we can easily correct 
the output frequency of the fountain for this effect with an accuracy of $5 \times 10^{-17}$ or better.

We first examine four frequency shifts for which we make explicit correction to the output frequency of the clock. These are the only significant corrections made to the clock and they are all in the realm of "planned" biases

Zeeman shift. In order to define a quantization axis for the atoms, a small $\left(\approx 10^{-7} \mathrm{~T}\right)$ magnetic field is applied to the atoms. This field (known for historical reasons as the $\mathrm{C}$-Field, although generally in a fountain there is neither an A nor a B field) is typically generated with a solenoid inside a magnetic shield package. This solenoid produces a homogeneous $(\approx 1 \%$ or better $)$ magnetic field parallel to the atoms' trajectory. As can be seen in Fig. 3, the magnetic field slightly distorts

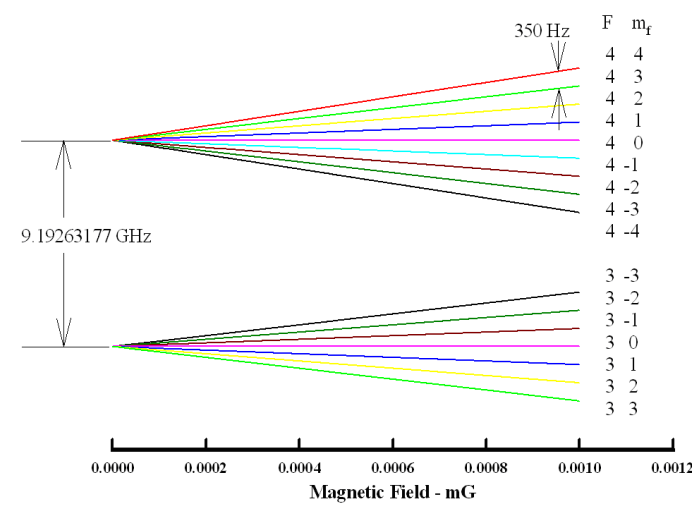

Fig. 3. The hyperfine splitting of the cesium atom as a function of applied magnetic field. As can be seen the $|3,0\rangle \leftrightarrow|4, p m 1\rangle$ transition is shifted only slightly, while the $|3,1\rangle \leftrightarrow|4,1\rangle$ transition changes rapidly with applied magnetic field. We can therefore use the $|3,1\rangle \leftrightarrow|4,1\rangle$ transition as a "magnetometer" to measure the magnetic field and use this information to correct the slight shift of the $|3,0\rangle \leftrightarrow|4,0\rangle$ transition.

the hyperfine structure of the atom. Because the frequency shift on the $|3,0\rangle \leftrightarrow$ $|4,0\rangle$ transition is insensitive to magnetic field to first order, the residual sensitivity is quadratic and is about $42.7 \mathrm{~Hz} / \mathrm{mT}^{2}$. The frequency shift on the $|3, \pm 1\rangle \leftrightarrow$ $|4, \pm 1\rangle$ transition is both much larger, $70.1 \mathrm{kHz} / \mathrm{mT}$, and linear in the magnetic field. We use this $|3, \pm 1\rangle \leftrightarrow|4, \pm 1\rangle$ transition to calibrate the magnetic field; that is, we measure the frequency difference between the $|3,0\rangle \leftrightarrow|4,0\rangle$ transition and the $|3, \pm 1\rangle \leftrightarrow|4,0\rangle$ transition. We then use this frequency difference to infer the frequency shift on the $|3,0\rangle \leftrightarrow|4,0\rangle$ transition. The much larger frequency shift on the $|3, \pm 1\rangle \leftrightarrow|4, \pm 1\rangle$ transition allows for a very precise calibration of the magnetic field and hence a very precise correction of the frequency shift of the $|3,0\rangle \leftrightarrow|4,0\rangle$ transition. The general philosophy of (a) using the atoms themselves to measure any frequency shifts whenever possible (thus measuring the effect in exactly the same environment as seen by the atoms in normal operation), and (b), seeking 
some sort of enhancement of the effect to be measured in order to "leverage" the precision of the measurement, is used whenever possible in this type of experiment.

Collision shifts. Cesium has long been known [7] to have a collision-induced frequency shift. This shift causes a frequency shift and in some fountains is the dominating uncertainty of the fountain. In NIST-F1 we have spent a considerable amount of time minimizing this shift and, having minimized it, then calibrating it. We minimize the shift using two distinct techniques. First, because the shift is linear in the density of the launched cesium sample, we use an extended optical molasses that gives a cigar shaped sample (with the long axis vertical). The sample has a radius of about $0.5 \mathrm{~cm}$ and a length of about $3 \mathrm{~cm}$, giving it a volume of about $2 \mathrm{~cm}^{3}$, larger by a factor of 10 than that typically used. Second, we use a particular optical geometry for the laser-cooling ( $\operatorname{lin} \perp \operatorname{lin}$ ) that allows us to get atom temperatures much lower $(T<500 \mathrm{nK})$ than the $\approx 2 \mu \mathrm{K}$ typically achieved using $\sigma^{+} \| \sigma^{-}$molasses. These low temperatures mean that a much larger fraction of the atoms that we launch contribute to the signal at the end of the ballistic flight thus allowing us to launch fewer atoms in the first place while still achieving a given $\mathrm{S} / \mathrm{N}$ ratio. The problem with higher temperatures at launch is that the launched atoms all contribute to the collision frequency shift even if they do not manage to find their way back to the detection zone to contribute to the $\mathrm{S} / \mathrm{N}$ ratio. In NIST-F1 the typical collision frequency shift is only $\delta f / f=5 \times 10^{-16}$, with an uncertainty of $1.5 \times 10^{-16}$. Because the collision frequency shift is not the dominant systematic uncertainty in NIST-F1, we work to reduce this uncertainty until it is small compared to others (notably blackbody), and we are reasonable confident that we can reduce the uncertainty associated with this shift to the $5 \times 10^{-17}$ level in NIST-F2, where we expect it to be among the dominant shifts.

Blackbody shift. In 1982 [8] it was observed that the room temperature blackbody radiation field within an atomic clock causes relatively large (by present day standards, in 1983 the shift was considered small) frequency shifts, on the order of $\delta f / f=2 \times 10^{-14}$ at room temperature. After several years of discussion it was decided to amend the definition of the second of the time to state "This definition refers to a cesium atom at rest at a temperature of $0 \mathrm{~K}$ ". We therefore must correct the output frequency of the fountain for the blackbody radiation field. The applicable formula is

$$
\frac{f_{T}-f_{0 \mathrm{~K}}}{f_{0 \mathrm{~K}}}=-1.711 \times 10^{-14}\left(\frac{T}{300}\right)^{4}\left(1+0.014\left(\frac{T}{300}\right)^{2}\right),
$$

where $T$ is the temperature of the radiation field that the atoms "see" within the standard during their ballistic flight. This temperature is typically taken as the temperature of the apparatus, however stray radiation entering the standard (through the windows that allow optical access in particular) can seriously affect the radiation temperature within the standard. While in NIST-F1 we control the temperature of the standard with a precision of millikelvins, we (perhaps overly) 
conservatively estimate the uncertainty of the radiation field to be equivalent to a $1 \mathrm{~K}$ uncertainty in the temperature $(320 \mathrm{~K})$ of the standard, resulting in an uncertainty of the blackbody bias of $2.8 \times 10^{-16}$.

Gravitational redshift. While the fountain produces proper time within its laboratory, all clocks are subject to frequency shifts due to both velocity (time dilation) and the local gravitational potential. By agreement, the output of the primary frequency standards are "corrected" as if they were located on an isopotential of the gravitational field known as the reference geoid. (The reference geoid is approximately mean sea level, which is a reasonable first approximation to an equipotential). The frequency of a clock is shifted by about $\delta f / f=+10^{-16} / \mathrm{m}$ of height above the geoid. Because Boulder, CO (where NIST-F1 is located) is some $1650 \mathrm{~m}$ above the geoid, the correction for this effect is the largest single correction made to NIST-F1. However, because of the high quality of gravitational mapping available, we know the height of NIST-F1 relative to the geoid with an uncertainty of $\approx 30 \mathrm{~cm}$, resulting in a negligible uncertainty on the clock frequency of $3 \times 10^{-17}$.

Unplanned biases, as previously mentioned, are also a constant source of concern (as well as irritation!) and experimentation. We very briefly discuss several of these in order to illustrate the "flavor" of these effects and our ability to control/eliminate them.

Fluorescent light shift. Stray light from the laser used to cool and detect the atoms entering the standard when the atoms are undergoing the Ramsey spectroscopy can cause large frequency shifts. The magnitude of these shifts can be estimated by noting that the saturation intensity is around $1 \mathrm{~mW} / \mathrm{cm}^{2}$ while the line width of the transition is of order $5 \mathrm{MHz}$. These combine to give an order of magnitude shift of $\delta f / f \approx 10^{-15} / \mathrm{fW}$, and since the typical laser intensity is of order of milliwatts it can be seen that the scattered light must be attenuated by much more than $120 \mathrm{~dB}$. In NIST-F1 the lasers are in a different room from the standard and are coupled into the standard with optical fibers. Before the light reaches the fibers it passes through two mechanical shutters with yet another shutter on the standard itself. By selectively removing these shutters we have shown that the fluorescent light shift in NIST-F1 should be less than $\delta f / f=10^{-20}$.

The shifts discussed above are a small subset of possible shifts. More complete discussions can be found in $[2,3]$. When all known possible shifts have been analyzed and/or measured, an accuracy budget is developed. An example from a recent NIST-F1 evaluation is shown in Table. The table lists the physical effect, the frequency bias associated with it, and the uncertainty of the bias. These uncertainties are conventionally combined in quadrature to reflect the systematic uncertainty of the standard. The collision shift (spin-exchange shift) uncertainty is included in the type $A$ uncertainty for obscure reasons that have to do with the way it is measured. The statistical uncertainty and the systematic uncertainty are then combined (again in quadrature) to give the total uncertainty associated with 
TABLE

The list of known frequency biases for NIST-F1. This includes both the magnitude of the biases as well as the uncertainty of each individual contribution to the final uncertainty. Units are fractional frequency, ${ }^{*} 10^{-15}$.

\begin{tabular}{l|c|c}
\hline \hline Physical effect & Bias & Type $B$ uncertainty \\
\hline gravitational red shift & +179.95 & 0.03 \\
\hline second-order Zeeman & +180.91 & 0.025 \\
\hline blackbody & -22.84 & 0.28 \\
\hline microwave amplitude shift & -0.05 & 0.15 \\
\hline spin exchange (low density) & $(-0.32)^{*}$ & $(0.17)^{*}$ \\
\hline AC Zeeman (heaters) & 0.05 & 0.05 \\
\hline cavity pulling & 0.02 & 0.02 \\
\hline Rabi pulling & $10^{-4}$ & $10^{-4}$ \\
\hline Ramsey pulling & $10^{-4}$ & $10^{-4}$ \\
\hline Majorana transitions & 0.02 & 0.02 \\
\hline fluorescence light shift & $10^{-5}$ & $10^{-5}$ \\
\hline cavity phase (distributed) & 0.02 & 0.02 \\
\hline second-order Doppler & 0.02 & 0.02 \\
\hline DC Stark effect & 0.02 & 0.02 \\
\hline background gas collisions & $10^{-3}$ & $10^{-3}$ \\
\hline Bloch-Siegert & $10^{-4}$ & $10^{-4}$ \\
\hline RF spectral purity & $3 \times 10^{-3}$ & $3 \times 10^{-3}$ \\
\hline integrator offset & 0 & 0.01 \\
\hline total type B standard uncertainty & 0.33
\end{tabular}

the fountain at the time of the report. For a typical recent report (Feb 2007) the total uncertainty of the fountain was of

$$
\frac{\delta f}{f}=5 \times 10^{-16},
$$

of which roughly half of the uncertainty is purely statistical and the other half a result of biases as shown in Table. Clearly, even with infinite run time, where the statistical uncertainty would be reduced to something approaching zero, the total uncertainty of the fountain would be only marginally improved to $\delta f / f=3.3 \times 10^{-16}$. This is primarily due to the blackbody frequency shift in our room-temperature apparatus which leads to an uncertainty of $\delta f / f \approx 3 \times 10^{-16}$.

\section{NIST-F2}

A glance at Table, the list of known frequency biases and their uncertainties for NIST-F1, shows that the dominant uncertainty is the blackbody shift at $320 \mathrm{~K}$. 
Worse yet, there is some controversy about the real size of the shift at room temperature. We have therefore built a new cesium fountain (NIST-F2) in which the Ramsey cavity and drift zone are at a temperature of $77 \mathrm{~K}$. As shown in Eq. (1), the blackbody shift scales (to lowest order) as $T^{4}$, so that the reduction from $320 \mathrm{~K}$ to $77 \mathrm{~K}$ reduces this shift by a factor of about 300 . This strong reduction in the effect lowers the size of the bias to $\delta f / f \approx 8 \times 10^{-17}$ ! The quality of the knowledge required for the correction of the bias is reduced as well, and therefore we believe that the blackbody shift will cease to be an issue in the error budget. We project that NIST-F2 should have a systematic uncertainty no greater than $\delta f / f \approx 1 \times 10^{-16}$.

\section{Acknowledgments}

We are very happy to acknowledge to valuable comments and suggestions which greatly improved this manuscript from our colleagues Paul Kunz, Stefania Romisch, Tom O'Brian, Mike Lombardi, and David Smith.

Contribution of the U.S. Government - not subject to U.S. Copyright.

\section{References}

[1] J.C. Bergquist, S.R. Jefferts, D.J. Wineland, Phys. Today 54, 37 (2001).

[2] S.R. Jefferts, J.H. Shirley, T.E. Parker, T.P. Heavner, D.M. Meekhof, C.W. Nelson, F. Levi, G. Costanzo, A. DeMarchi, R.E. Drullinger, L. Hollberg, W.D. Lee, F.L. Walls, Metrologia 39, 321 (2002).

[3] T.P. Heavner, S.R. Jefferts, E.A. Donley, J.H. Shirley, T.E. Parker, Metrologia 42, 411 (2005).

[4] T.E. Parker, S.R. Jefferts, T.P. Heavner, E.A. Donley, Metrologia 42, 423 (2005).

[5] J.A. Barnes, Basic Concepts of Precise Time and Frequency, N.B.S. Monograph, Ch. 1.

[6] W.M. Itano, J.C. Bergquist, J.J. Bollinger, J.M. Gilligan, D.J. Heinzen, F.L. Moore, M.G. Raizen, D.J. Wineland, Phys. Rev. A 47, 3554 (1993).

[7] E. Tiesinga, B.J. Verhaar, H.T.C. Stoof, D. vanBragt, Phys. Rev. A 45, 2671 (1992).

[8] W.M. Itano, L.L. Lewis, D.J. Wineland, Phys. Rev. A 25, 1233 (1982).

[9] S.R. Jefferts, J.H. Shirley, N. Ashby, E.A. Burt, G.J. Dick, IEEE T. Ultrason. 52, 2314 (2005).

[10] J.H. Shirley, F. Levi, T.P. Heavner, D. Calonico, D. Yu, S.R. Jefferts, IEEE T. Ultrason. 53, 2376 (2006).

[11] F. Levi, J.H. Shirley, T.P. Heavner, D. Yu, S.R. Jefferts, IEEE T. Ultrason. 53, 1584 (2006). 